\title{
Generalized Fractional Integral Inequalities for Continuous Random Variables
}

\author{
Abdullah Akkurt, Zeynep Kaçar, and Hüseyin Yildirim \\ Department of Mathematics, Faculty of Science and Arts, University of Kahramanmaraş Sütçü İmam, 46000 Kahramanmaraş, Turkey \\ Correspondence should be addressed to Abdullah Akkurt; abdullahmat@gmail.com
}

Received 5 October 2014; Accepted 9 December 2014

Academic Editor: Z. D. Bai

Copyright (C) 2015 Abdullah Akkurt et al. This is an open access article distributed under the Creative Commons Attribution License, which permits unrestricted use, distribution, and reproduction in any medium, provided the original work is properly cited.

Some generalized integral inequalities are established for the fractional expectation and the fractional variance for continuous random variables. Special cases of integral inequalities in this paper are studied by Barnett et al. and Dahmani.

\section{Introduction}

Integral inequalities play a fundamental role in the theory of differential equations, functional analysis, and applied sciences. Important development in this theory has been achieved in the last two decades. For these, see [1-8] and the references therein. Moreover, the study of fractional type inequalities is also of vital importance. Also see [9-13] for further information and applications. The first one is given in [14]; in their paper, using Korkine identity and Holder inequality for double integrals, Barnett et al. established several integral inequalities for the expectation $E(X)$ and the variance $\sigma^{2}(X)$ of a random variable $X$ having a probability density function (p.d.f.) $f:[a, b] \rightarrow \mathbb{R}^{+}$. In [15-17] the authors presented new inequalities for the moments and for the higher order central moments of a continuous random variable. In $[17,18]$ Dahmani and Miao and Yang gave new upper bounds for the standard deviation $\sigma(X)$, for the quantity $\sigma^{2}(X)+(t-E(X))^{2}, t \in[a, b]$, and for the $L^{p}$ absolute deviation of a random variable $X$. Recently, Anastassiou et al. [9] proposed a generalization of the weighted Montgomery identity for fractional integrals with weighted fractional Peano kernel. More recently, Dahmani and Niezgoda [17, 19] gave inequalities involving moments of a continuous random variable defined over a finite interval. Other papers dealing with these probability inequalities can be found in [20-22].

In this paper, we introduce new concepts on "generalized fractional random variables." We obtain new generalized integral inequalities for the generalized fractional dispersion and the generalized fractional variance functions of a continuous random variable $X$ having the probability density function (p.d.f.) $f:[a, b] \rightarrow \mathbb{R}^{+}$by using these concepts. Our results are extension of $[12,14,17]$.

\section{Preliminaries}

Definition 1 (see [23]). Let $f \in L^{1}[a, b]$. The RiemannLiouville fractional integrals $J_{a^{+}}^{\alpha} f(x)$ and $J_{b^{-}}^{\alpha} f(x)$ of order $\alpha \geq 0$ are defined by

$$
\begin{aligned}
& J_{a^{+}}^{\alpha}[f(x)]=\frac{1}{\Gamma(\alpha)} \int_{a}^{x}(x-t)^{\alpha-1} f(t) d t \quad x>a, \\
& J_{b^{-}}^{\alpha}[f(x)]=\frac{1}{\Gamma(\alpha)} \int_{x}^{b}(t-x)^{\alpha-1} f(t) d t \quad x<b,
\end{aligned}
$$

respectively, where $\Gamma(\alpha)=\int_{0}^{\infty} e^{-u} u^{\alpha-1} d u$ is Gamma function and $J_{a^{+}}^{0} f(x)=J_{b^{-}}^{0} f(x)=f(x)$.

We give the following properties for the $J_{a^{+}}^{\alpha}$ :

$$
\begin{gathered}
J_{a^{+}}^{\alpha} J_{a^{+}}^{\beta}[f(t)]=J_{a^{+}}^{\alpha+\beta}[f(t)], \quad \alpha \geq 0, \beta \geq 0, \\
J_{a^{+}}^{\alpha} J_{a^{+}}^{\beta}[f(t)]=J_{a^{+}}^{\beta} a_{a^{+}}^{\alpha}[f(t)], \quad \alpha \geq 0, \beta \geq 0 .
\end{gathered}
$$


Definition 2 (see [24]). Consider the space $L_{p, k}(a, b)(k \geq$ $0,1 \leq p<\infty)$ of those real-valued Lebesgue measurable functions $f$ on $[a, b]$ for which

$$
\begin{array}{r}
\|f\|_{L_{p, k(a, b)}}=\left(\int_{a}^{b}|f(x)|^{p} x^{k} d x\right)^{1 / p}<\infty \\
1 \leq p<\infty, \quad k \geq 0 .
\end{array}
$$

Definition 3 (see [24]). Consider the space $X_{c}^{p}(a, b)(c \in$ $R, 1 \leq p<\infty)$ of those real-valued Lebesgue measurable functions $f$ on $[a, b]$ for which

$$
\begin{array}{r}
\|f\|_{X_{c}^{p}}=\left(\int_{a}^{b}\left|x^{c} f(x)\right|^{p} \frac{d x}{x}\right)^{1 / p}<\infty, \\
(1 \leq p<\infty, c \in R)
\end{array}
$$

and for the case $p=\infty$

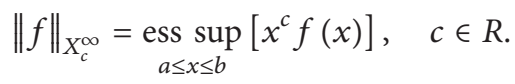

In particular, when $c=(k+1) / p(1 \leq p<\infty, k \geq 0)$ the space $X_{c}^{p}(a, b)$ coincides with the $L_{p, k}(a, b)$-space and also if we take $c=(1 / p)(1 \leq p<\infty)$ the space $X_{c}^{p}(a, b)$ coincides with the classical $L^{p}(a, b)$-space.

Definition 4 (see [24]). Let $f \in L_{1, k}[a, b]$. The generalized Riemann-Liouville fractional integrals $J_{a^{+}}^{\alpha, k} f(x)$ and $J_{b^{-}}^{\alpha, k} f(x)$ of orders $\alpha \geq 0$ and $k \geq 0$ are defined by

$J_{a^{+}}^{\alpha, k} f(x)=\frac{(k+1)^{1-\alpha}}{\Gamma(\alpha)} \int_{a}^{x}\left(x^{k+1}-t^{k+1}\right)^{\alpha-1} t^{k} f(t) d t \quad x>a$,

$$
J_{b^{-}}^{\alpha, k} f(x)=\frac{(k+1)^{1-\alpha}}{\Gamma(\alpha)} \int_{x}^{b}\left(t^{k+1}-x^{k+1}\right)^{\alpha-1} t^{k} f(t) d t \quad b>x .
$$

Here $\Gamma(\alpha)$ is Gamma function and $J_{a^{+}}^{0, k} f(x)=J_{b^{-}}^{0, k} f(x)=$ $f(x)$. Integral formulas (7) and (8) are called right generalized Riemann-Liouville integral and left generalized RiemannLiouville fractional integral, respectively.

Definition 5. The fractional expectation function of orders $\alpha \geq 0$ and $k \geq 0$, for a random variable $X$ with a positive p.d.f. $f$ defined on $[a, b]$, is defined as

$$
\begin{aligned}
E_{X, \alpha}(t) & :=J_{a^{+}}^{\alpha, k}[t f(t)] \\
& =\frac{(k+1)^{1-\alpha}}{\Gamma(\alpha)} \int_{a}^{t}\left(t^{k+1}-\tau^{k+1}\right)^{\alpha-1} \tau^{k+1} f(\tau) d \tau, \\
& a<t \leq b .
\end{aligned}
$$

In the same way, we define the fractional expectation function of $X-E(X)$ by what follows.
Definition 6. The fractional expectation function of orders $\alpha \geq 0, k \geq 0$, and $a<t \leq b$, for a random variable $X-E(X)$, is defined as

$$
\begin{aligned}
& E_{X-E(X), \alpha}(t) \\
& \qquad \begin{array}{l}
:=\frac{(k+1)^{1-\alpha}}{\Gamma(\alpha)} \int_{a}^{t}\left(t^{k+1}-\tau^{k+1}\right)^{\alpha-1} \\
\quad \times(\tau-E(X)) \tau^{k} f(\tau) d \tau,
\end{array}
\end{aligned}
$$

where $f:[a, b] \rightarrow \mathbb{R}^{+}$is the p.d.f. of $X$.

For $t=b$, we introduce the following concept.

Definition 7. The fractional expectation of orders $\alpha \geq 0, a<$ $t \leq b$, and $k \geq 0$, for a random variable $X$ with a positive p.d.f. $f$ defined on $[a, b]$, is defined as

$$
E_{X, \alpha}=\frac{(k+1)^{1-\alpha}}{\Gamma(\alpha)} \int_{a}^{b}\left(b^{k+1}-\tau^{k+1}\right)^{\alpha-1} \tau^{k+1} f(\tau) d \tau .
$$

For the fractional variance of $X$, we introduce the following two definitions.

Definition 8. The fractional variance function of orders $\alpha \geq 0$, $a<t \leq b$, and $k \geq 0$, for a random variable $X$ having a p.d.f. $f:[a, b] \rightarrow \mathbb{R}^{+}$, is defined as

$$
\begin{aligned}
\sigma_{X, \alpha}^{2} & :=J_{a^{+}}^{\alpha, k}\left[(t-E(X))^{2} f(t)\right] \\
& =\frac{(k+1)^{1-\alpha}}{\Gamma(\alpha)} \int_{a}^{t}\left(t^{k+1}-\tau^{k+1}\right)^{\alpha-1}(\tau-E(X))^{2} \tau^{k} f(\tau) d \tau,
\end{aligned}
$$

where $E(X):=\int_{a}^{b} \tau f(\tau) d \tau$ is the classical expectation of $X$.

Definition 9. The fractional variance of order $\alpha \geq 0$, for a random variable $X$ with a p.d.f. $f:[a, b] \rightarrow \mathbb{R}^{+}$, is defined as

$$
\begin{aligned}
\sigma_{X, \alpha}^{2}=\frac{(k+1)^{1-\alpha}}{\Gamma(\alpha)} \int_{a}^{b} & \left(b^{k+1}-\tau^{k+1}\right)^{\alpha-1} \\
& \times(\tau-E(X))^{2} \tau^{k} f(\tau) d \tau .
\end{aligned}
$$

We give the following important properties.

(1) If we take $\alpha=1$ and $k=0$ in Definition 5, we obtain the classical expectation $E_{X, 1}=E(X)$.

(2) If we take $\alpha=1$ and $k=0$ in Definition 7, we obtain the classical variance $\sigma_{X, 1}^{2}=\sigma^{2}(X)=\int_{a}^{b}(\tau-$ $E(X))^{2} f(\tau) d \tau$.

(3) If we take $k=0$ in Definitions 5-9, we obtain Definitions 2.2-2.6 in [17].

(4) For $\alpha>0$, the p.d.f. $f$ satisfies $J^{\alpha}[f(b)]=(b-$ $a)^{\alpha-1} / \Gamma(\alpha)$.

(5) For $\alpha=1$, we have the well known property $J^{\alpha}[f(b)]=1$. 


\section{Main Results}

Theorem 10. Let $X$ be a continuous random variable having ap.d.f. $f:[a, b] \rightarrow \mathbb{R}^{+}$. Then

(a) for all $a<t \leq b, \alpha \geq 0$, and $k \geq 0$,

$$
\begin{aligned}
J_{a^{+}}^{\alpha, k}[f(t)] \sigma_{X, \alpha}^{2}(t)-\left(E_{X-E(X), \alpha}(t)\right)^{2} \\
\leq\|f\|_{\infty}^{2}\left[\frac{(k+1)^{1-\alpha}\left(t^{k+1}-a^{k+1}\right)^{\alpha}}{\Gamma(\alpha+1)} J_{a^{+}}^{\alpha, k}\left[t^{2 k+2}\right]\right. \\
\left.-\left(J_{a^{+}}^{\alpha, k}[t]\right)^{2}\right],
\end{aligned}
$$

provided that $f \in L_{\infty}[a, b]$;

(b) the inequality

$$
\begin{gathered}
J_{a^{+}}^{\alpha, k}[f(t)] \sigma_{X, \alpha}^{2}(t)-\left(E_{X-E(X), \alpha}(t)\right)^{2} \\
\leq \frac{1}{2}\left(t^{k+1}-a^{k+1}\right)^{2}\left(J_{a^{+}}^{\alpha, k}[t]\right)^{2}
\end{gathered}
$$

is also valid for all $a<t \leq b, \alpha \geq 0$, and $k \geq 0$.

Proof. Let us define the quantity for p.d.f. $g$ and $h$ :

$$
\begin{array}{r}
H(\tau, \rho):=(g(\tau)-g(\rho))(h(\tau)-h(\rho)) \\
\tau, \rho \in(a, t), \quad a<t \leq b, \quad \alpha \geq 0 .
\end{array}
$$

Taking a function $p:[a, b] \rightarrow \mathbb{R}^{+}$, multiplying (16) by $\left(\left(t^{k+1}\right.\right.$ $\left.\left.-\tau^{k+1}\right)^{\alpha-1} / \Gamma(\alpha)\right) p(\tau) \tau^{k}, \tau \in(a, t)$, and then integrating the resulting identity with respect to $\tau$ from $a$ to $t$, we have

$$
\begin{gathered}
\frac{(k+1)^{1-\alpha}}{\Gamma(\alpha)} \int_{a}^{t}\left(t^{k+1}-\tau^{k+1}\right)^{\alpha-1} p(\tau) H(\tau, \rho) \tau^{k} f(\tau) d \tau \\
=J_{a^{+}}^{\alpha, k}[p g h(t)]-h(\rho) J_{a^{+}}^{\alpha, k}[p g(t)] \\
\quad-g(\rho) J_{a^{+}}^{\alpha, k}[p h(t)]+g(\rho) h(\rho) J_{a^{+}}^{\alpha, k}[p(t)] .
\end{gathered}
$$

Similarly, multiplying (17) by $\left(\left(t^{k+1}-\rho^{k+1}\right)^{\alpha-1} / \Gamma(\alpha)\right) p(\rho) \rho^{k}$, $\rho \in(a, t)$, and integrating the resulting identity with respect to $\rho$ over $(a, t)$, we can write

$$
\begin{gathered}
\frac{(k+1)^{2-2 \alpha}}{\Gamma^{2}(\alpha)} \int_{a}^{t} \int_{a}^{t}\left(t^{k+1}-\tau^{k+1}\right)^{\alpha-1}\left(t^{k+1}-\rho^{k+1}\right)^{\alpha-1} \\
\quad \times p(\tau) p(\rho) H(\tau, \rho) \tau^{k} \rho^{k} f(\tau) d \tau d \rho \\
=2 J_{a^{+}}^{\alpha, k}[p(t)] J_{a^{+}}^{\alpha, k}[p g h(t)] \\
-2 J_{a^{+}}^{\alpha, k}[p g(t)] J_{a^{+}}^{\alpha, k}[p h(t)] .
\end{gathered}
$$

If, in (18), we take $p(t)=f(t)$ and $g(t)=h(t)=t^{k+1}-E(X)$, $t \in(a, b)$, then we have

$$
\begin{aligned}
& \frac{(k+1)^{2-2 \alpha}}{\Gamma^{2}(\alpha)} \\
& \quad \times \int_{a}^{t} \int_{a}^{t}\left(t^{k+1}-\tau^{k+1}\right)^{\alpha-1}\left(t^{k+1}-\rho^{k+1}\right)^{\alpha-1} \\
& \quad \times f(\tau) f(\rho)\left(\tau^{k+1}-\rho^{k+1}\right)^{2} \tau^{k} \rho^{k} f(\tau) d \tau d \rho \\
& =2 J_{a^{+}}^{\alpha, k}[f(t)] J_{a^{+}}^{\alpha, k}\left[f(t)\left(t^{k+1}-E(X)\right)^{2}\right] \\
& \quad-2\left[J_{a^{+}}^{\alpha, k} f(t)\left(t^{k+1}-E(X)\right)\right]^{2} .
\end{aligned}
$$

On the other hand, we have

$$
\begin{aligned}
& \frac{(k+1)^{2-2 \alpha}}{\Gamma^{2}(\alpha)} \\
& \times \int_{a}^{t} \int_{a}^{t}\left(t^{k+1}-\tau^{k+1}\right)^{\alpha-1}\left(t^{k+1}-\rho^{k+1}\right)^{\alpha-1} \\
& \quad \times f(\tau) f(\rho)\left(\tau^{k+1}-\rho^{k+1}\right)^{2} \tau^{k} \rho^{k} f(\tau) d \tau d \rho \\
& \leq\|f\|_{\infty}^{2}\left[\begin{array}{l}
2 \frac{(k+1)^{1-\alpha}\left(t^{k+1}-a^{k+1}\right)^{\alpha}}{\Gamma(\alpha+1)} J_{a^{+}}^{\alpha, k}\left[t^{2 k+2}\right] \\
\left.-2\left(J_{a^{+}}^{\alpha, k}[t]\right)^{2}\right] .
\end{array}\right.
\end{aligned}
$$

Thanks to (19) and (20), we obtain part (a) of Theorem 10. For part (b), we have

$$
\begin{aligned}
& \frac{(k+1)^{2-2 \alpha}}{\Gamma^{2}(\alpha)} \\
& \quad \times \int_{a}^{t} \int_{a}^{t}\left(t^{k+1}-\tau^{k+1}\right)^{\alpha-1}\left(t^{k+1}-\rho^{k+1}\right)^{\alpha-1} \\
& \quad \times f(\tau) f(\rho)\left(\tau^{k+1}-\rho^{k+1}\right)^{2} \tau^{k} \rho^{k} f(\tau) d \tau d \rho \\
& \leq \sup _{\tau, \rho \in[a, t]}\left|\left(\tau^{k+1}-\rho^{k+1}\right)\right|^{2}\left[J_{a^{+}}^{\alpha, k} f(t)\right]^{2} \\
& =\left(t^{k+1}-a^{k+1}\right)^{2}\left[J_{a^{+}}^{\alpha, k} f(t)\right]^{2} .
\end{aligned}
$$

Then, by (19) and (21), we get the desired inequality (14).

We give also the following corollary.

Corollary 11. Let $X$ be a continuous random variable with a p.d.f. $f$ defined on $[a, b]$. Then 
(i) if $f \in L_{\infty}[a, b]$, then for any $\alpha \geq 0$ and $k \geq 0$, one has

$$
\begin{aligned}
& \frac{\left(b^{k+1}-a^{k+1}\right)^{(\alpha-1)}}{\Gamma(\alpha)} \sigma_{X, \alpha}^{2}-E_{X, \alpha}^{2} \\
& \leq\|f\|_{\infty}^{2}\left[\frac{\left(b^{k+1}-a^{k+1}\right)^{2 \alpha+2}}{\Gamma(\alpha+1) \Gamma(\alpha+3)}-\left(\frac{\left(b^{k+1}-a^{k+1}\right)^{\alpha+1}}{\Gamma(\alpha+1)}\right)^{2}\right] ;
\end{aligned}
$$

(ii) the inequality

$$
\frac{\left(b^{k+1}-a^{k+1}\right)^{(\alpha-1)}}{\Gamma(\alpha)} \sigma_{X, \alpha}^{2}-E_{X, \alpha}^{2} \leq \frac{1}{2}\left[\frac{\left(b^{k+1}-a^{k+1}\right)^{2 \alpha}}{\Gamma^{2}(\alpha)}\right]
$$

$$
\text { is also valid for any } \alpha \geq 0 \text { and } k \geq 0 \text {. }
$$

Remark 12. (r1) Taking $\alpha=1$ and $k=0$ in (i) of Corollary 11, we obtain the first part of Theorem 1 in [14].

(r2) Taking $\alpha=1$ and $k=0$ in (ii) of Corollary 11, we obtain the last part of Theorem 1 in [14].

We will further generalize Theorem 10 by considering two fractional positive parameters.

Theorem 13. Let $X$ be a continuous random variable having a p.d.f. $f:[a, b] \rightarrow \mathbb{R}^{+}$. Then one has the following.

(a) For all $a<t \leq b, \alpha \geq 0, \beta \geq 0$, and $k \geq 0$,

$$
\begin{gathered}
J_{a^{+}}^{\alpha, k}[f(t)] \sigma_{X, \beta}^{2}(t)+J_{a^{+}}^{\beta, k}[f(t)] \sigma_{X, \alpha}^{2}(t) \\
-2\left(E_{X-E(X), \alpha}(t)\right)\left(E_{X-E(X), \beta}(t)\right) \\
\leq\|f\|_{\infty}^{2}\left[\frac{(k+1)^{1-\alpha}\left(t^{k+1}-a^{k+1}\right)^{\alpha}}{\Gamma(\alpha+1)} J_{a^{+}}^{\beta, k}\left[t^{2 k+2}\right]\right] \\
+\|f\|_{\infty}^{2}\left[\frac{(k+1)^{1-\beta}\left(t^{k+1}-a^{k+1}\right)^{\beta}}{\Gamma(\beta+1)} J_{a^{+}}^{\alpha, k}\left[t^{2 k+2}\right]\right. \\
\left.-2\left(J_{a^{+}}^{\alpha, k}[t]\right)\left(J_{a^{+}}^{\beta, k}[t]\right)\right],
\end{gathered}
$$

where $f \in L_{\infty}[a, b]$.

(b) The inequality

$$
\begin{gathered}
J_{a^{+}}^{\alpha, k}[f(t)] \sigma_{X, \beta}^{2}(t)+J_{a^{+}}^{\beta, k}[f(t)] \sigma_{X, \alpha}^{2}(t) \\
-2\left(E_{X-E(X), \alpha}(t)\right)\left(E_{X-E(X), \beta}(t)\right) \\
\leq\left(t^{k+1}-a^{k+1}\right)^{2}\left(J_{a^{+}}^{\alpha, k}[t]\right)\left(J_{a^{+}}^{\beta, k}[t]\right)
\end{gathered}
$$

is also valid for any $a<t \leq b, \alpha \geq 0, \beta \geq 0$, and $k \geq 0$.
Proof. Using (15), we can write

$$
\begin{aligned}
& \frac{(k+1)^{2-\alpha-\beta}}{\Gamma(\alpha) \Gamma(\beta)} \int_{a}^{t} \int_{a}^{t}\left(t^{k+1}-\tau^{k+1}\right)^{\alpha-1}\left(t^{k+1}-\rho^{k+1}\right)^{\alpha-1} \\
& \times p(\tau) p(\rho) H(\tau, \rho) \tau^{k} \rho^{k} f(\tau) d \tau d \rho \\
& =J_{a^{+}}^{\alpha, k}[p(t)] J_{a^{+}}^{\beta, k}[p g h(t)]+J_{a^{+}}^{\beta, k}[p(t)] J_{a^{+}}^{\alpha, k}[p g h(t)] \\
& \quad-J_{a^{+}}^{\alpha, k}[p h(t)] J_{a^{+}}^{\beta, k}[p g(t)] \\
& \quad-J_{a^{+}}^{\beta, k}[p h(t)] J_{a^{+}}^{\alpha, k}[p g(t)] .
\end{aligned}
$$

Taking $p(t)=f(t)$ and $g(t)=h(t)=t^{k+1}-E(X), t \in(a, b)$, in the above identity, yields

$$
\begin{aligned}
& \frac{(k+1)^{2-\alpha-\beta}}{\Gamma(\alpha) \Gamma(\beta)} \\
& \times \int_{a}^{t} \int_{a}^{t}\left(t^{k+1}-\tau^{k+1}\right)^{\alpha-1}\left(t^{k+1}-\rho^{k+1}\right)^{\alpha-1} \\
& \quad \times p(\tau) p(\rho)\left(\tau^{k+1}-\rho^{k+1}\right)^{2} \tau^{k} \rho^{k} f(\tau) d \tau d \rho \\
& =J_{a^{+}}^{\alpha, k}[f(t)] J_{a^{+}}^{\beta, k}\left[f(t)\left(t^{k+1}-E(X)\right)^{2}\right] \\
& \quad+J_{a^{+}}^{\beta, k}[f(t)] J_{a^{+}}^{\alpha, k}\left[f(t)\left(t^{k+1}-E(X)\right)^{2}\right] \\
& \quad-2 J_{a^{+}}^{\alpha, k}\left[f(t)\left(t^{k+1}-E(X)\right)\right] J_{a^{+}}^{\beta, k} \\
& \quad \times\left[f(t)\left(t^{k+1}-E(X)\right)\right] .
\end{aligned}
$$

We have also

$$
\begin{gathered}
\frac{(k+1)^{2-\alpha-\beta}}{\Gamma(\alpha) \Gamma(\beta)} \\
\times \int_{a}^{t} \int_{a}^{t}\left(t^{k+1}-\tau^{k+1}\right)^{\alpha-1}\left(t^{k+1}-\rho^{k+1}\right)^{\alpha-1} \\
\quad \times p(\tau) p(\rho)\left(\tau^{k+1}-\rho^{k+1}\right)^{2} \tau^{k} \rho^{k} f(\tau) d \tau d \rho \\
\leq\|f\|_{\infty}^{2}\left[\frac{(k+1)^{1-\alpha}\left(t^{k+1}-a^{k+1}\right)^{\alpha}}{\Gamma(\alpha+1)} J_{a^{+}}^{\beta, k}\left[t^{2 k+2}\right]\right. \\
+\frac{(k+1)^{1-\beta}\left(t^{k+1}-a^{k+1}\right)^{\beta}}{\Gamma(\beta+1)} J_{a^{+}}^{\alpha, k}\left[t^{2 k+2}\right] \\
\left.-2\left(J_{a^{\alpha}}^{\alpha, k}[t]\right)\left(J_{a^{+}}^{\beta, k}[t]\right)\right] .
\end{gathered}
$$

Thanks to (27) and (28), we obtain (a). 
To prove (b), we use the fact that $\sup _{\tau, \rho \in[a, t]} \mid\left(\tau^{k+1}-\right.$ $\left.\rho^{k+1}\right)\left.\right|^{2}=\left(t^{k+1}-a^{k+1}\right)^{2}$. We obtain

$$
\begin{aligned}
& \frac{(k+1)^{2-\alpha-\beta}}{\Gamma(\alpha) \Gamma(\beta)} \\
& \quad \times \int_{a}^{t} \int_{a}^{t}\left(t^{k+1}-\tau^{k+1}\right)^{\alpha-1}\left(t^{k+1}-\rho^{k+1}\right)^{\alpha-1} \\
& \quad \times f(\tau) f(\rho)\left(\tau^{k+1}-\rho^{k+1}\right)^{2} \tau^{k} \rho^{k} f(\tau) d \tau d \rho \\
& \leq\left(t^{k+1}-a^{k+1}\right)^{2}\left(J_{a^{+}}^{\alpha, k}[t]\right)\left(J_{a^{+}}^{\beta, k}[t]\right) .
\end{aligned}
$$

And, by (27) and (29), we get (25).

Remark 14. (r1) Applying Theorem 13 for $\alpha=\beta$, we obtain Theorem 10.

We give also the following fractional integral result.

Theorem 15. Let $f$ be the p.d.f. of $X$ on $[a, b]$. Then for all $a<$ $t \leq b, \alpha \geq 0$, and $k \geq 0$, one has

$$
\begin{array}{r}
J_{a^{+}}^{\alpha, k}[f(t)] \sigma_{X, \alpha}^{2}(t)-\left(E_{X-E(X), \alpha}(t)\right)^{2} \\
\leq \frac{1}{4}\left(b^{k+1}-a^{k+1}\right)^{2}\left(J_{a^{+}}^{\alpha, k}[t]\right)^{2} .
\end{array}
$$

Proof. Using Theorem 1 of [25], we can write

$$
\begin{gathered}
\left|J_{a^{+}}^{\alpha, k}[p(t)] J_{a^{+}}^{\alpha, k}\left[p g^{2}(t)\right]-\left(J_{a^{+}}^{\alpha, k}[p g(t)]\right)^{2}\right| \\
\leq \frac{1}{4}\left(J_{a^{+}}^{\alpha, k}[p(t)]\right)^{2}(M-m)^{2} .
\end{gathered}
$$

Taking $p(t)=f(t)$ and $g(t)=t^{k+1}-E(X), t \in(a, b)$, then $M=b^{k+1}-E(X)$ and $m=a^{k+1}-E(X)$. Hence, (30) allows us to obtain

$$
\begin{aligned}
0 \leq & J_{a^{+}}^{\alpha, k}[f(t)] J_{a^{+}}^{\alpha, k}\left[f(t)\left(t^{k+1}-E(X)\right)^{2}\right] \\
& -\left(J_{a^{+}}^{\alpha, k}\left[f(t)\left(t^{k+1}-E(X)\right)^{2}\right]\right)^{2} \\
\leq & \frac{1}{4}\left(J_{a^{+}}^{\alpha, k}[f(t)]\right)^{2}\left(b^{k+1}-a^{k+1}\right)^{2} .
\end{aligned}
$$

This implies that

$$
\begin{array}{r}
J_{a^{+}}^{\alpha, k}[f(t)] \sigma_{X, \alpha}^{2}(t)-\left(E_{X-E(X), \alpha}(t)\right)^{2} \\
\leq \frac{1}{4}\left(b^{k+1}-a^{k+1}\right)^{2}\left(J_{a^{+}}^{\alpha, k}[t]\right)^{2} .
\end{array}
$$

Theorem 15 is thus proved.

For $t=b$, we propose the following interesting inequality.
Corollary 16. Let $f$ be the p.d.f. of $X$ on $[a, b]$. Then for any $\alpha \geq 0$ and $k \geq 0$, one has

$$
\begin{gathered}
\frac{\left(b^{k+1}-a^{k+1}\right)^{(\alpha-1)}}{\Gamma(\alpha)} \sigma_{X, \alpha}^{2}-\left(E_{X-E(X), \alpha}(t)\right)^{2} \\
\leq \frac{1}{4 \Gamma^{2}(\alpha)}\left(b^{k+1}-a^{k+1}\right)^{2 \alpha} .
\end{gathered}
$$

Remark 17. Taking $\alpha=1$ in Corollary 16, we obtain Theorem 2 of [14].

We also present the following result for the fractional variance function with two parameters.

Theorem 18. Let $f$ be the p.d.f. of the random variable $X$ on $[a, b]$. Then for all $a<t \leq b, \alpha \geq 0, \beta \geq 0$, and $k \geq 0$, one has

$$
\begin{aligned}
J_{a^{+}}^{\alpha, k}[f(t)] \sigma_{X, \beta}^{2}(t)+J_{a^{+}}^{\beta, k}[f(t)] \sigma_{X, \alpha}^{2}(t) \\
+2\left(a^{k+1}-E(X)\right)\left(b^{k+1}-E(X)\right) \\
\times J_{a^{+}}^{\alpha, k}[f(t)] J_{a^{+}}^{\beta, k}[f(t)] \\
\leq\left(a^{k+1}+b^{k+1}-2 E(X)\right) \\
\quad \times\left(J_{a^{+}}^{\alpha, k}[f(t)]\left(E_{X-E(X), \beta}(t)\right)\right. \\
\left.\quad+J_{a^{+}}^{\beta, k}[f(t)]\left(E_{X-E(X), \alpha}(t)\right)\right) .
\end{aligned}
$$

Proof. Thanks to Theorem 4 of [25], we can state that

$$
\begin{aligned}
{\left[J_{a^{+}}^{\alpha, k}[p(t)] J_{a^{+}}^{\beta, k}\left[p g^{2}(t)\right]+J_{a^{+}}^{\beta, k}[p(t)] J_{a^{+}}^{\alpha, k}\left[p g^{2}(t)\right]\right.} \\
\left.-2 J_{a^{+}}^{\alpha, k}[p g(t)] J_{a^{+}}^{\beta, k}[p g(t)]\right]^{2} \\
\leq\left[\left(M J_{a^{+}}^{\alpha, k}[p(t)]-J_{a^{+}}^{\alpha, k}[p g(t)]\right)\right. \\
\times\left(J_{a^{+}}^{\beta, k}[p g(t)]-m J_{a^{+}}^{\beta, k}[p(t)]\right) \\
+\left(J_{a^{+}}^{\beta, k}[p g(t)]-m J_{a^{+}}^{\beta, k}[p(t)]\right) \\
\left.\times\left(M J_{a^{+}}^{\beta, k}[p(t)]-J_{a^{+}}^{\beta, k}[p g(t)]\right)\right]^{2} .
\end{aligned}
$$

In (35), we take $p(t)=f(t)$ and $g(t)=t^{k+1}-E(X), t \in(a, b)$. We obtain

$$
\begin{aligned}
& {\left[J_{a^{+}}^{\alpha, k}[f(t)] J_{a^{+}}^{\beta, k}\left[f(t)\left(t^{k+1}-E(X)\right)^{2}\right]\right.} \\
& \quad+J_{a^{+}}^{\beta, k}[f(t)] J_{a^{+}}^{\alpha, k}\left[f(t)\left(t^{k+1}-E(X)\right)^{2}\right] \\
& \left.\quad-2 J_{a^{+}}^{\alpha, k}\left[f(t)\left(t^{k+1}-E(X)\right)\right] J_{a^{+}}^{\beta, k}\left[f(t)\left(t^{k+1}-E(X)\right)\right]\right]^{2}
\end{aligned}
$$




$$
\begin{aligned}
\leq & {\left[\left(M J_{a^{+}}^{\alpha, k}[f(t)]-J_{a^{+}}^{\alpha, k}\left[f(t)\left(t^{k+1}-E(X)\right)\right]\right)\right.} \\
& \times\left(J_{a^{+}}^{\beta, k}\left[f(t)\left(t^{k+1}-E(X)\right)\right]-m J_{a^{+}}^{\beta, k}[f(t)]\right) \\
& +\left(J_{a^{+}}^{\alpha, k}\left[f(t)\left(t^{k+1}-E(X)\right)\right]-m J_{a^{+}}^{\alpha, k}[f(t)]\right) \\
& \left.\times\left(M J_{a^{+}}^{\beta, k}[f(t)]-J_{a^{+}}^{\beta, k}\left[f(t)\left(t^{k+1}-E(X)\right)\right]\right)\right]^{2} .
\end{aligned}
$$

Combining (27) and (37) and taking into account the fact that the left-hand side of (27) is positive, we get

$$
\begin{aligned}
J_{a^{+}}^{\alpha, k}[ & f(t)] J_{a^{+}}^{\beta, k}\left[f(t)\left(t^{k+1}-E(X)\right)^{2}\right] \\
& +J_{a^{+}}^{\beta, k}[f(t)] J_{a^{+}}^{\alpha, k}\left[f(t)\left(t^{k+1}-E(X)\right)^{2}\right] \\
& -2 J_{a^{+}}^{\alpha, k}\left[f(t)\left(t^{k+1}-E(X)\right)\right] J_{a^{+}}^{\beta, k}\left[f(t)\left(t^{k+1}-E(X)\right)\right] \\
\leq & \left(M J_{a^{+}}^{\alpha, k}[f(t)]-J_{a^{+}}^{\alpha, k}\left[f(t)\left(t^{k+1}-E(X)\right)\right]\right) \\
& \times\left(J_{a^{+}}^{\beta, k}\left[f(t)\left(t^{k+1}-E(X)\right)\right]-m J_{a^{+}}^{\beta, k}[f(t)]\right) \\
& +\left(J_{a^{+}}^{\alpha, k}\left[f(t)\left(t^{k+1}-E(X)\right)\right]-m J_{a^{+}}^{\alpha, k}[f(t)]\right) \\
& \times\left(M J_{a^{+}}^{\beta, k}[f(t)]-J_{a^{+}}^{\beta, k}\left[f(t)\left(t^{k+1}-E(X)\right)\right]\right) .
\end{aligned}
$$

Therefore,

$$
\begin{array}{r}
J_{a^{+}}^{\alpha, k}[f(t)] J_{a^{+}}^{\beta, k}\left[f(t)\left(t^{k+1}-E(X)\right)^{2}\right] \\
+J_{a^{+}}^{\beta, k}[f(t)] J_{a^{+}}^{\alpha, k}\left[f(t)\left(t^{k+1}-E(X)\right)^{2}\right] \\
\leq M\left(J_{a^{+}}^{\alpha, k}[f(t)]\left(E_{X-E(X), \beta}(t)\right)\right. \\
\left.+J_{a^{+}}^{\beta, k}\left[f(t)\left(E_{X-E(X), \alpha}(t)\right)\right]\right) \\
+m\left(J_{a^{+}}^{\alpha, k}[f(t)]\left(E_{X-E(X), \beta}(t)\right)\right. \\
\left.+J_{a^{+}}^{\beta, k}\left[f(t)\left(E_{X-E(X), \alpha}(t)\right)\right]\right) .
\end{array}
$$

Substituting the values of $m$ and $M$ in (33), then a simple calculation allows us to obtain (35). Theorem 18 is thus proved.

To finish, we present to the reader the following corollary.

Corollary 19. Let $f$ be the p.d.f. of $X$ on $[a, b]$. Then for all $a<t \leq b, \alpha \geq 0$, and $k \geq 0$, the inequality

$$
\begin{gathered}
\sigma_{X, \alpha}^{2}(t)+\left(a^{k+1}-E(X)\right)\left(b^{k+1}-E(X)\right) J_{a^{+}}^{\alpha, k}[f(t)] \\
\leq\left(a^{k+1}+b^{k+1}-2 E(X)\right) E_{X-E(X), \alpha}(t)
\end{gathered}
$$

is valid.

\section{Conflict of Interests}

The authors declare that there is no conflict of interests regarding the publication of this paper.

\section{References}

[1] N. S. Barnett, P. Cerone, S. S. Dragomir, and J. Roumeliotis, "Some inequalities for the expectation and variance of a random variable whose PDF is n-time differentiable," Journal of Inequalities in Pure and Applied Mathematics, vol. 1, no. 2, article 21, 2000.

[2] P. L. Chebyshev, "Sur les expressions approximatives des integrales definies par les autres prises entre les mêmes limites," Proceedings of the Mathematical Society of Kharkov, vol. 2, pp. 9398, 1882.

[3] S. S. Dragomir, "A generalization of Grussís inequality in inner product spaces and applications," Journal of Mathematical Analysis and Applications, vol. 237, no. 1, pp. 74-82, 1999.

[4] D. S. Mitrinović, J. E. Pečarić, and A. M. Fink, Classical and New Inequalities in Analysis, vol. 61 of Mathematics and its Applications, Kluwer Academic Publishers Group, Dordrecht, Dordrecht, The Netherlands, 1993.

[5] B. G. Pachpatte, "On multidimensional Gruss type integral inequalities," Journal of Inequalities in Pure and Applied Mathematics, vol. 32, pp. 1-15, 2002.

[6] F. Qi, A. J. Li, W. Z. Zhao, D. W. Niu, and J. Cao, "Extensions of several integral inequalities," Journal of Inequalities in Pure and Applied Mathematics, vol. 7, no. 3, pp. 1-6, 2006.

[7] F. Qi, "Several integral inequalities," Journal of Inequalities in Pure and Applied Mathematics, vol. 1, no. 2, pp. 1-9, 2000.

[8] M. Z. Sarikaya, N. Aktan, and H. Yildirim, "On weighted Čebyšev-Grüss type inequalities on time scales," Journal of Mathematical Inequalities, vol. 2, no. 2, pp. 185-195, 2008.

[9] G. A. Anastassiou, M. R. Hooshmandasl, A. Ghasemi, and F. Moftakharzadeh, "Montgomery identities for fractional integrals and related fractional inequalities," Journal of Inequalities in Pure and Applied Mathematics, vol. 10, no. 4, pp. 1-6, 2009.

[10] G. A. Anastassiou, Fractional Differentiation Inequalities, Springer Science, 2009.

[11] S. Belarbi and Z. Dahmani, "On some new fractional integral inequalities," Journal of Inequalities in Pure and Applied Mathematics, vol. 10, no. 3, pp. 1-12, 2009.

[12] Z. Dahmani, "New inequalities in fractional integrals," International Journal of Nonlinear Science, vol. 9, no. 4, pp. 493-497, 2010.

[13] Z. Dahmani, "On Minkowski and Hermite-Hadamad integral inequalities via fractional integration," Annals of Functional Analysis, vol. 1, no. 1, pp. 51-58, 2010.

[14] N. S. Barnett, P. Cerone, S. S. Dragomir, and J. Roumeliotis, "Some inequalities for the dispersion of a random variable whose PDF is defined on a finite interval," Journal of Inequalities in Pure and Applied Mathematics, vol. 2, no. 1, pp. 1-18, 2001.

[15] P. Kumar, "Moment inequalities of a random variable defined over a finite interval," Journal of Inequalities in Pure and Applied Mathematics, vol. 3, no. 3, pp. 1-24, 2002.

[16] P. Kumar, "Inequalities involving moments of a continuous random variable defined over a finite interval," Computers and Mathematics with Applications, vol. 48, no. 1-2, pp. 257-273, 2004 
[17] Z. Dahmani, "Fractional integral inequalities for continuous random variables," Malaya Journal of Matematik, vol. 2, no. 2, pp. 172-179, 2014.

[18] Y. Miao and G. Yang, "A note on the upper bounds for the dispersion," Journal of Inequalities in Pure and Applied Mathematics, vol. 8, no. 3, pp. 1-13, 2007.

[19] M. Niezgoda, "New bounds for moments of continuous random variables," Computers \& Mathematics with Applications, vol. 60, no. 12, pp. 3130-3138, 2010.

[20] A. M. Acu, F. Sofonea, and C. V. Muraru, "Gruss and Ostrowski type inequalities and their applications," Scientific Studies and Research: Series Mathematics and Informatics, vol. 23, no. 1, pp. 5-14, 2013.

[21] T. F. Mori, "Sharp inequalities between centered moments," Journal of Mathematical Analysis and Applications, vol. 10, no. 4, pp. 1-19, 2009.

[22] R. Sharma, S. Devi, G. Kapoor, S. Ram, and N. S. Barnett, "A brief note on some bounds connecting lower order moments for random variables defined on a finite interval," International Journal of Theoretical \& Applied Sciences, vol. 1, no. 2, pp. 83-85, 2009.

[23] S. G. Samko, A. A. Kilbas, and O. I. Marichev, Fractional Integrals and Derivatives, Theory and Applications, Gordon and Breach, Yverdon, Switzerland, 1993.

[24] H. Yıldırım and Z. Kırtay, "Ostrowski inequality for generalized fractional integral and related inequalities," Malaya Journal of Matematik, vol. 2, no. 3, pp. 322-329, 2014.

[25] Z. Dahmani and L. Tabharit, "On weighted Gruss type inequalities via fractional integrals," Journal of Advanced Research in Pure Mathematics, vol. 2, no. 4, pp. 31-38, 2010. 


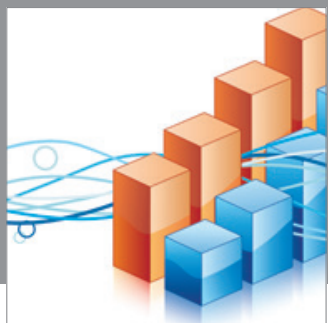

Advances in

Operations Research

mansans

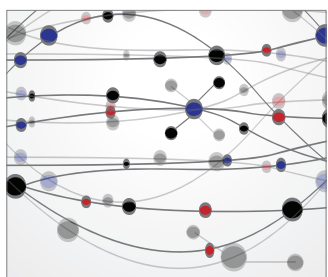

The Scientific World Journal
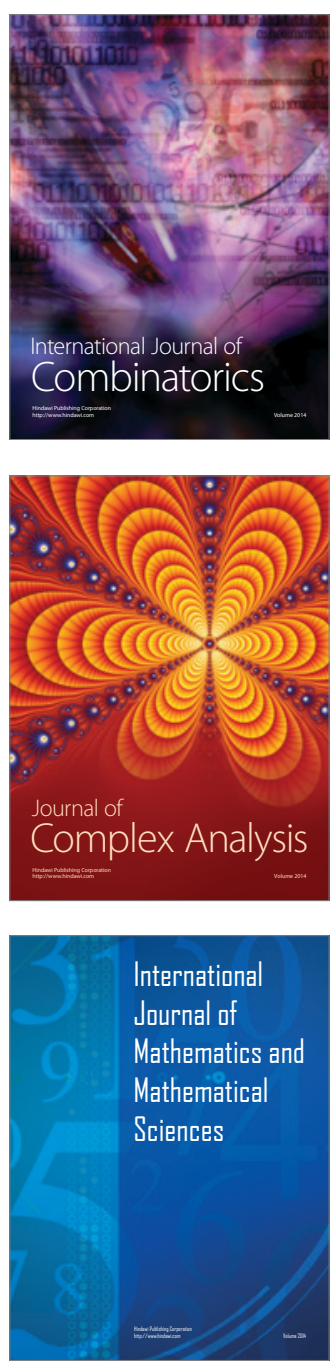
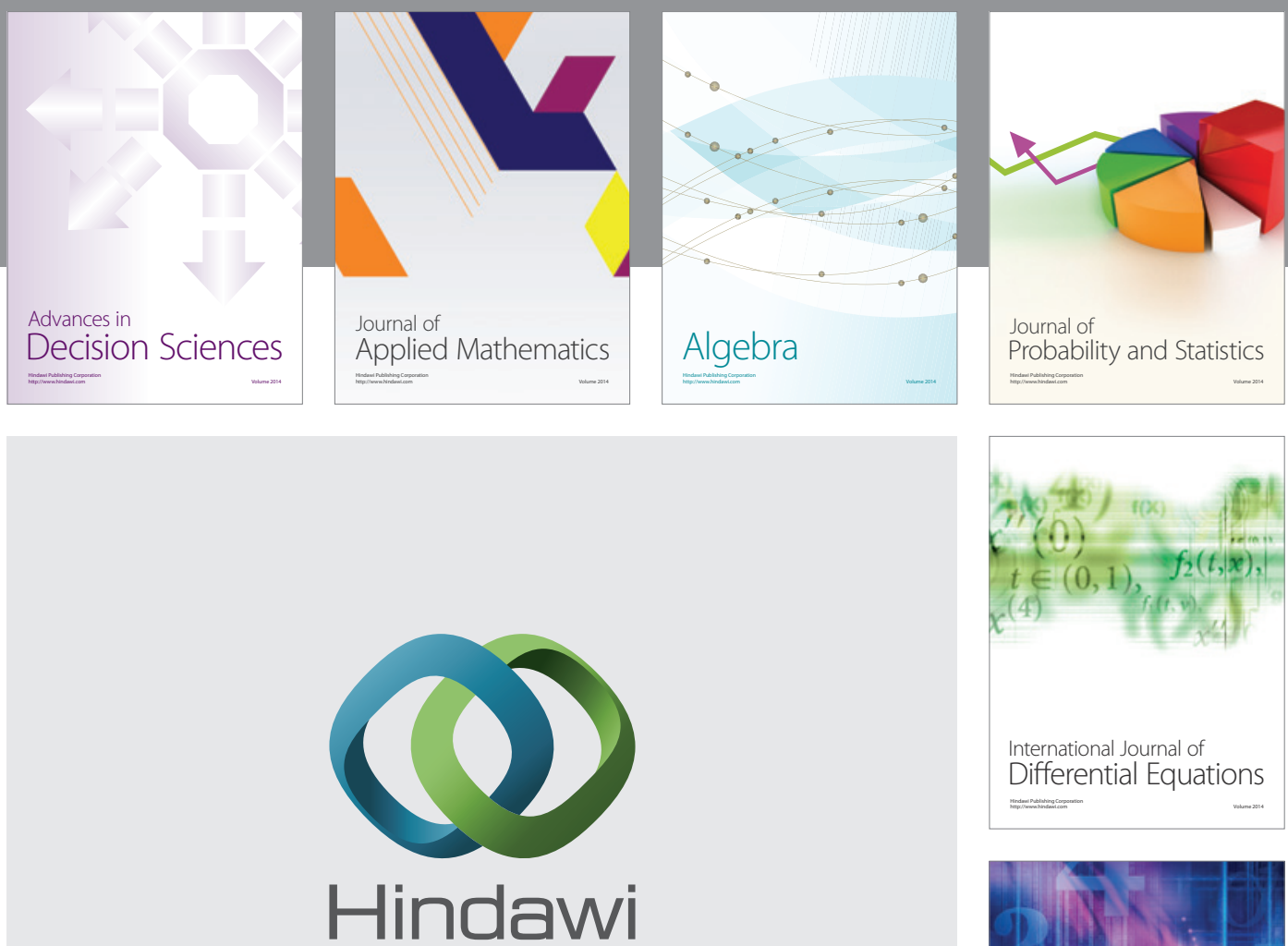

Submit your manuscripts at http://www.hindawi.com
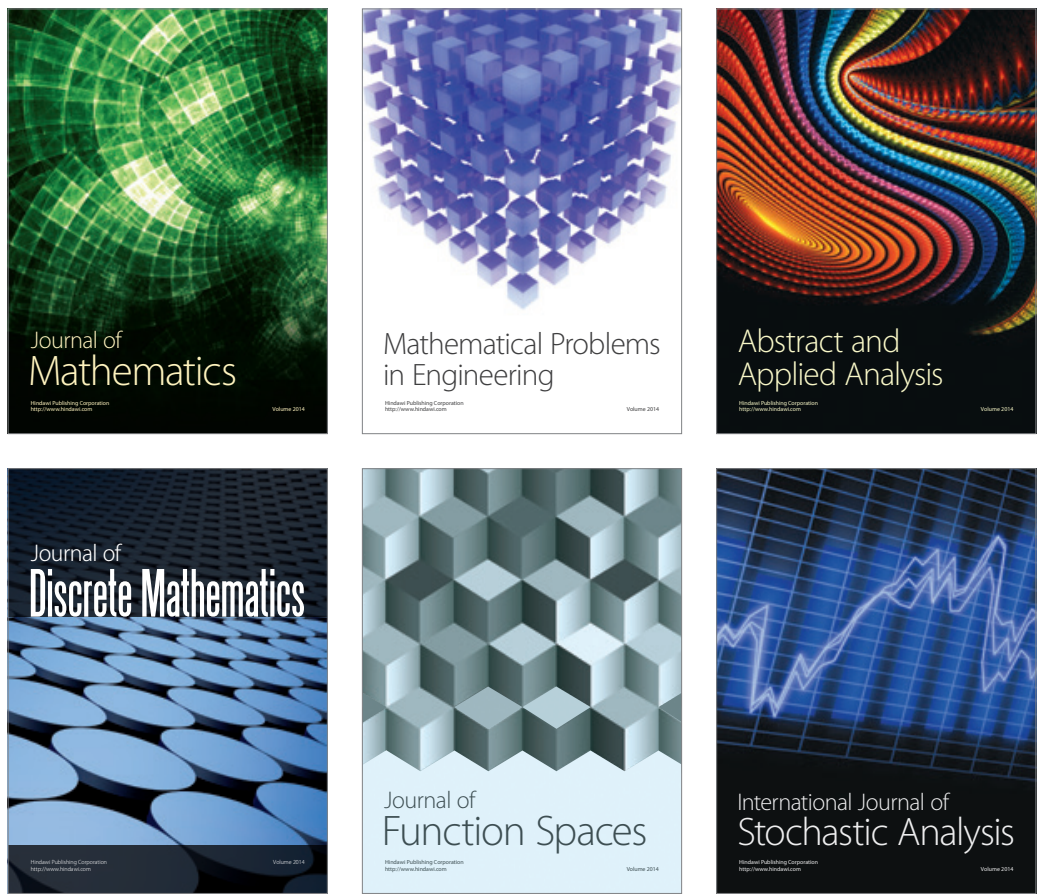

Journal of

Function Spaces

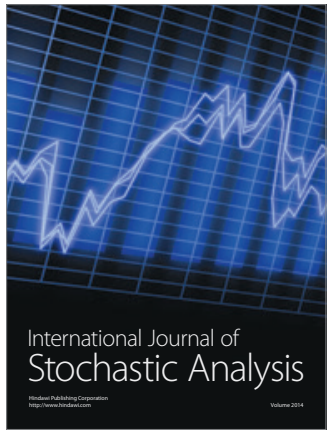

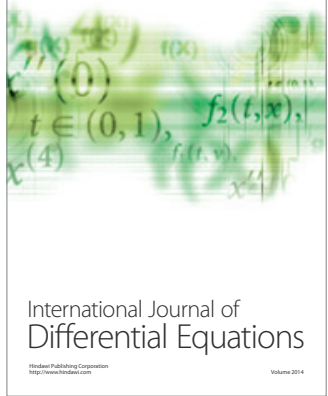
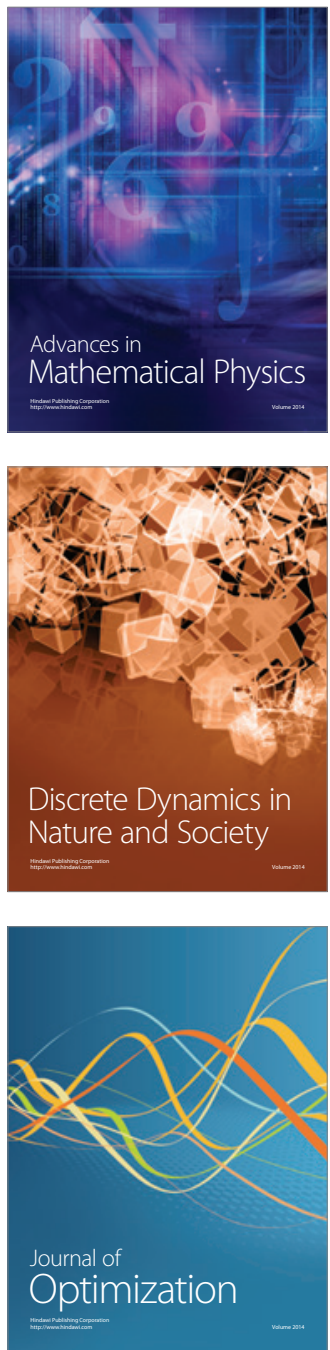